\title{
Candido Ghiotti : une figure emblématique du professeur de français (Italie, fin XIX ${ }^{\mathrm{e}}$ siècle)
}

\author{
Marie-France Merger
}

\section{(2) OpenEdition \\ 1 Journals}

\section{Édition électronique}

URL : https://journals.openedition.org/dhfles/1100

DOI : $10.4000 /$ dhfles. 1100

ISSN : 2221-4038

Éditeur

Société Internationale pour l'Histoire du Français Langue Étrangère ou Seconde

Édition imprimée

Date de publication : 1 décembre 2005

Pagination : 75-90

ISSN : 0992-7654

Référence électronique

Marie-France Merger, « Candido Ghiotti : une figure emblématique du professeur de français (Italie, fin XIX siècle) », Documents pour l'histoire du français langue étrangère ou seconde [En ligne], 35 | 2005, mis en ligne le 14 août 2011, consulté le 27 mai 2021. URL : http://journals.openedition.org/dhfles/1100 ; DOI : https://doi.org/10.4000/dhfles. 1100

Ce document a été généré automatiquement le 27 mai 2021.

(C) SIHFLES 


\title{
Candido Ghiotti : une figure emblématique du professeur de français (Italie, fin XIX ${ }^{\mathrm{e}}$ siècle)
}

\author{
Marie-France Merger
}

1 Si nous évoquons encore aujourd'hui le nom de Ghiotti, des personnes ayant étudié le français en Italie dans les années 1950 se rappellent bien souvent leur dictionnaire bilingue à la couverture rouge qui a jalonné leurs études et leurs exercices de thème et version. Si nous remontons dans le temps et que nous allions consulter les catalogues, nous nous apercevrons que Ghiotti n'est pas seulement un lexicographe mais aussi un auteur de grammaires, de dialogues, d'une chrestomathie et qu'il a publié de nombreux ouvrages à la fin du XIX ${ }^{\mathrm{e}}$ siècle.

\section{Sa carrière}

Qui était donc ce Candido Ghiotti dont le nom est resté célèbre jusqu"aux années 1970 ? Son dossier aux Archives de la Pubblica Istruzione à Rome ${ }^{1}$ qui ne concerne que la période 1860-1880, nous informe qu'il est né à Turin en 1841 et qu'il a suivi un «Cours complet" spécial correspondant à la «licenza» (le baccalauréat) de l'Institut Technique. Il a également suivi un cours privé de latin et de rhétorique; il a obtenu un brevet qui l'a habilité à l'enseignement du français et de l'anglais et il est en possession de la " patente normale superiore ». Le début de sa carrière d'enseignant est placé sous le signe de la précarité, de la mobilité territoriale et de la pluralité des disciplines enseignées car il n'est pas encore titulaire. Son premier poste est à Alessandria pour l'année 1859-1860 où il est professeur de français et d'anglais; l'année suivante, il est professeur d'anglais au Regio Istituto Tecnico de Turin puis à la Scuola Tecnica de Varallo dans la province de Vercelli (Piémont). Pendant deux ans (de 1862 à 1864) il enseigne dans les Marches à Pesaro ; il y fera un remplacement à la Scuola Tecnica. Il revient à Alessandria de septembre 1864 à 1871, où il est à la fois professeur de français à la Scuola Tecnica et professeur d'anglais à l'Institut technique. En 1872, il est muté à Frosinone dans le Latium, toujours dans une Scuola Tecnica; c'est là qu'en 1880, il est 
enfin nommé directeur de cette école et professeur titulaire de français; nous ignorons combien de temps il est resté sur ce poste car nous ne possédons pas de renseignements sur la période suivante, néanmoins les préfaces de sa grammaire et de son dictionnaire bilingue nous indiquent qu'en 1884, il était professeur de français à la Regia Scuola Tecnica Germano Sommeiller de Turin, une école dont il est devenu le directeur et où il a fini sa carrière.

Les lettres que Ghiotti adresse aux autorités supérieures font état bien souvent des difficultés matérielles qu'il rencontre dans son métier d'enseignant non titulaire. Ainsi dans une lettre adressée au ministre de l'Instruction Publique et datée du 17 août 1861, il demande un "poste fixe pour le français dans une école technique de l'État» (ibid.). Dans une autre lettre toujours adressée au ministre, le 24 octobre 1863, Ghiotti - qui enseigne à Pesaro - se plaint de son «tenue stipendio » (maigre traitement) de 1280 lires et supplie le ministre de lui accorder un traitement pour les classes supérieures d'un montant de 1440 lires. À l'appui de sa requête il joint une lettre du «provveditore agli studi » (une sorte d'inspecteur d'Académie) de la province de Pesaro et Urbino, datée du 27 octobre 1863 où ce dernier défend ce "jeune professeur [qui] s'est toujours montré plein de zèle, honnête et intelligent » (ibid.). Lorsqu'il est nommé à Frosinone, il sera chargé d'un remplacement de "computisteria», c'est-à-dire de comptabilité pendant l'année scolaire 1873-1874: la rémunération de Ghiotti se fera attendre puisque son dossier contient d'une part une lettre datée du 25 juillet $1873 \mathrm{du}$ provveditore agli studi, un certain Carbone, lequel demande une «equa remunerazione " pour ce professeur remplaçant; de l'autre, mais... un an plus tard, celle du Prefetto Presidente des écoles de la province, qui répète qu'il faut accorder un " equo compenso » à Candido Ghiotti qui a fait preuve de beaucoup de compétence et a obtenu de «bons résultats » (ibid.) de la part de ses élèves. Ce n'est cependant pas dans cette matière que Ghiotti va faire carrière.

4 Rappelons qu'il est l'auteur de nombreux ouvrages : une chrestomathie publiée en 1866, une grammaire dont la première édition remonte à 1868, une refonte des dialogues de Morand et un dictionnaire bilingue dont les éditions se prolongeront jusqu'en 1973 ; en outre, avec son collègue Giustino Dogliani, il publie de nombreuses anthologies, un manuel de correspondance commerciale ainsi que des recueils d'exercices (cf. Bibliographie). Il m'est apparu intéressant de centrer mon attention sur la figure de ce professeur en tant que défenseur d'une méthode.

\section{Sa grammaire : un début prometteur}

5 Le 22 mars 1868, alors qu'il est professeur à la Regia Scuola Tecnica d'Alessandria, il adresse au Consiglio Superiore di Pubblica Istruzione une lettre (ibid.) de présentation de son ouvrage dont le titre est le suivant: Grammatica ragionata della lingua francese ; cette grammaire est publiée la même année par l'éditeur Paolo Ragazzone et porte un sous-titre sans équivoque : Nuovo corso di lezioni teorico-pratiche dettate col confronto della Lingua Italiana e coll'Analisi Filologica, c'est-à-dire " Nouveau cours de leçons théoriques et pratiques dictées avec la comparaison avec l'italien et l'analyse philologique ». Cette grammaire est précédée d'une préface où l'auteur illustre brièvement sa démarche qu'il qualifie de «metodo filologico comparato " (méthode philologique comparée. $1868: 3$ ).

6 La première idée que Ghiotti développe dans cette Préface est que pour enseigner le français, il faut partir de la connaissance de la langue maternelle, l'italien. Il s'agit d'un 
véritable défi car l'Unité italienne vient d'être réalisée depuis peu et la Péninsule est encore un pays très dialectophone malgré la loi Casati ${ }^{2}$ qui a organisé tout le système scolaire et notamment l'enseignement secondaire, rendant obligatoire l'étude du français dans les écoles techniques. Ghiotti évolue dans un climat culturel, social, politique fortement marqué par l'honneur national, un « paysage » que Carla Pellandra a déjà tracé dans des études précédentes ${ }^{3}$. Cette idée d'une langue nationale sera développée avec plus de détermination dans la Préface de la nouvelle édition de sa grammaire en 1875 : l'auteur écrit en effet qu'à présent, en Italie, « la vie politique et civile s'est réveillée » (Ghiotti 1875 : IV). Il est heureux que les grammairiens italiens se soient enfin aperçus qu'ils possèdent une langue reproduisant «très fidèlement et en grande partie, les formes orthographiques et grammaticales de la langue jumelle ", c'est-à-dire le français. Dans sa grammaire Ghiotti montrera donc l'analogie orthographique qui lie le français et l'italien et qui, d'après lui, aide les élèves dans l'apprentissage de la langue étrangère; il donne entre autres l'exemple des " combinazioni omotone » au et eau : autre, auguste, faute sont facilement expliqués par les formes italiennes altro, augusto, fallo qui contiennent le $a$ du français, tandis que l'orthographe de marteau, beau, cerveau, s'explique par le e présent dans les mots italiens correspondants : martello, bello, cervello. Un autre exemple peut être celui des consonnes finales non prononcées en français de coup, blanc, tout, bas, nom, sourd qui viennent de l'italien, colpo, bianco, tutto, basso, nome, sordo (1884:258), même si le choix d'un mot comme nom avec le $\mathrm{m}$ final formant une voyelle nasale ne me semble pas très pertinent.

7 Il ajoute, toujours dans sa Préface, qu'il était temps que le sentiment national se réveille et que l'on antépose l'étude de l'italien (" la lingua patria ») à celle des autres langues". Donc, si la mode, le goût, l'industrie ne réussissent pas à se libérer de l'influence transalpine, au moins que l'enseignement de la langue s'« émancipe » de l'influence française, tel est le souhait de Ghiotti. Il critique donc l'habitude enracinée chez les Italiens d'enseigner le français avec des systèmes et des grammaires servant aux élèves français pour étudier leur propre langue. Ces ouvrages ne sont pas adaptés aux élèves italiens car ils sont encombrés de définitions de grammaire générale, de répétitions de règles communes aux deux langues sans aucune comparaison entre le français et l'italien. Il est évident que Ghiotti pense aux grammaires de Lhomond ou de Noël et Chapsal qui étaient éditées aussi en Italie ${ }^{5}$. La méthode de Ghiotti est au contraire comparative : l'italien servant de base à l'enseignement du français, cela permettra aux élèves de saisir et de comprendre les rapports qui existent entre les deux langues.

Cette méthode est également rationnelle : en enseignant, Ghiotti s'est rendu compte que les élèves veulent « connaître les causes » des règles qui leur sont enseignées, c'est pourquoi il a conçu un cours de "lezioni ragionate» (leçons raisonnées) qu'il a regroupées dans un "cahier de grammaire $»^{6}:$ c'est là l'origine de sa méthode et de sa grammaire car, déclare-t-il, l'édition de 1868 est le reflet fidèle de ce cahier de grammaire (qu'il dicte en classe), si l'on excepte les notes qui remplacent les observations et les explications verbales.

9 Il est évident que Ghiotti critique indirectement les méthodes de Ahn et d'Ollendorff, des méthodes intuitives et imitatives, qui jouissaient d'une grande faveur auprès des professeurs et qui faisaient apprendre une langue étrangère comme on apprend la langue maternelle, négligeant totalement les facultés rationnelles des élèves. Ses critiques seront beaucoup plus explicites dans sa Relazione alla Commissione Ministeriale, 
intorno all'insegnamento della Lingua francese nelle scuole italiane; il s'agit d'un rapport qui sera publié par le ministère de l'Instruction publique et qu'il avait envoyé au député Boselli le 2 octobre $1879^{7}$; il l'introduira ensuite dans la Préface de l'édition de sa grammaire en 1884.

Ghiotti y répète les mêmes concepts en insistant sur le fait que les aptitudes à retenir, à se souvenir de la part des élèves vont en s'affaiblissant au fil des années, alors que leurs facultés rationnelles se développent : il faut donc s'adresser à l'intelligence et laisser à la mémoire la tâche de retenir « la partie pour ainsi dire, matérielle de la langue que l'on veut apprendre " (Ghiotti 1884: V). La grammaire qu'il se propose d'enseigner, écrit-il, "même si elle est raisonnée, ne contiendra pas d'observations historicophilologiques, mais au contraire, sous la forme de lois générales, les résultats de ces observations" (ibid). C'est aux professeurs que revient la tâche d'approfondir les relations philologiques d'une langue avec les autres et de communiquer aux élèves les résultats pratiques de leurs recherches. Ces résultats se présenteront sous la forme d'une série d'avertissements qui serviront de raisonnement aux règles; ainsi, comme son expérience le prouve, poursuit-il, les apprenants auront une idée exacte du caractère de la langue, ces avertissements leur servant de "gymnastique intellectuelle ».

11 Pour illustrer sa méthode, Ghiotti présente l'exemple du futur : remontant au latin, il signale que Cicéron avait eu recours à l'auxiliaire habere uni à l'infinitif d'un verbe pour exprimer une action dans le futur (habeo ad te scribere). Le peuple a généralisé cette tendance et a composé avec habeo un futur qui a remplacé la forme classique. Avec le temps, cette forme composée devient un temps simple qui donnera scriverò (scriver ho), scriverai (scriver hai)... en italien et j'écrirai, tu écriras... en français. Certes, il est inutile d'exposer ces observations à des élèves des écoles techniques, ajoute-t-il, elles peuvent cependant intéresser les élèves des lycées ou les étudiants à l'université; il suffira de dire aux premiers que l'on forme le futur tout simplement avec l'infinitif du verbe uni aux formes de l'indicatif présent de l'auxiliaire avoir.

12 En ce qui concerne l'enseignement de la prononciation, là aussi Ghiotti utilise le système philologique comparé des deux langues; je ne citerai qu'un exemple, celui de la prononciation de $\mathbf{x}$. «La lettre $x$ peut avoir trois sons différents: de gs (doux), de cs (dur), de ss. La comparaison entre les deux langues suffit pour les indiquer de manière précise. Les grammaires moins empiriques disent que $\mathbf{x}$ prend le premier son lorsqu'il se trouve entre deux voyelles; le deuxième lorsqu'il suit une consonne; le troisième dans quelques mots que l'on cite comme des anomalies ». En fait, cette règle n'explique pas le son cs dans les mots comme "fixe, rixe, axe, luxe, sexe, etc, où il se trouve entre deux voyelles». Voici donc la règle énoncée par Ghiotti à partir de la comparaison entre les deux langues : « $\mathbf{x}$ entre deux voyelles donne le son cs, s'il est représenté par ss dans le mot italien correspondant et lorsqu'il suit une consonne; gs dans le cas contraire, ainsi les mots qui se présentent comme des exceptions se verront regroupés dans la règle générale» (Ghiotti 1884: VIII). En réalité, on trouve le son cs - pour reprendre la terminologie de Ghiotti - lorsque $\mathbf{x}$ précède une consonne comme dans les mots texte, textile et non pas quand il la suit ; signalons que Ghiotti avait bien énoncé 'sa' règle de prononciation dans l'édition de 1875 (13).

13 En suivant ce système, la distinction rigoureuse entre syntaxe et grammaire élémentaire, déclare l'auteur, n'est plus possible, puisque les règles qui concernent la concordance et la disposition des parties du discours doivent être signalées 
alternativement, et au fur et à mesure qu'elles sont nécessaires pour la traduction de l'italien en français. De ce fait l'ouvrage n'est plus partagé en Grammaire et en Syntaxe mais en Lexicologie, et Syntaxe élémentaire, en Syntaxe et Lexicologie supérieure.

À la fin de chaque leçon de grammaire, Ghiotti introduit deux traductions en français, deux thèmes. Le premier est une traduction interlinéaire à laquelle l'auteur ajoute les mots qui ne se rapportent pas aux règles exposées précédemment, et cela d'une part, " pour ne pas distraire l'esprit des élèves dans la traduction qu'on leur donne », tout de suite après l'explication des règles; d'autre part pour offrir des exercices qui puissent être faits oralement comme préparation à la seconde traduction qui se fera par écrit. Le second thème est proposé « sans la partie matérielle du lexique et cela pour habituer les élèves à la recherche, au choix logique des mots, car l'utilisation du dictionnaire a aussi ses difficultés» (ibid.: [VII]) qu'il faut surmonter par la pratique. Ainsi il donne des conseils aux professeurs et aux élèves : avant de faire les exercices de traduction, il faut faire attention aux lettres $s$. indiquant un substantif, $a$. un adjectif, $m$. le masculin et $f$. le féminin, qui permettent de distinguer les homonymes italiens, leur signification, leur valeur intrinsèque, "les différentes acceptions des mots indiqués par le dictionnaire italien-français, correspondant au mot italien que l'on y a cherché. Si, par exemple, le mot mezzo peut se traduire par demi, moitié, centre, moyen, etc., la recherche de chacun de ces mots dans le dictionnaire français-italien fera connaître la valeur différente de ceux-ci, et indiquera lequel doit être choisi pour représenter la signification propre du mot mezzo que l'on veut traduire». Ghiotti se rend bien compte que ce travail a peu d'attraits pour les élèves de « mediocre volontà » mais, d'après lui, c'est « la seule voie qui peut conduire à des résultats satisfaisants » $(1875: 85)$.

Dans les éditions postérieures à 1890 , date de la publication de son dictionnaire scolaire, Ghiotti se fait de la publicité : les difficultés des exercices de traduction seront aplanies grâce aux indications qu'il a introduites dans son Vocabolario scolastico delle lingue italiana e francese (1898: 63) qui est le résumé de son ouvrage en deux volumes, le Nuovo Vocabolario comparativo delle lingue italiana e francese dont la publication date de 1883.

17 Ghiotti ne s'est pas limité à rédiger une grammaire ; en décembre 1883, sort le premier numéro de La lingua francese nelle scuole secondarie d'Italia, une revue qu'il crée avec son collègue Giustino Dogliani ; il s'agit d'un périodique qui est envoyé gratuitement aux professeurs de français des écoles secondaires et dont le sous-titre, encore une fois, est sans équivoque: Periodico di Filologia comparata, Grammatica ragionata e Didattica. Ici encore Ghiotti défend sa méthode "à la fois comparative, rationnelle et pratique » et reprend les idées qu'il a déjà exposées dans les différentes éditions de sa grammaire en insistant sur la naissance d'une nouvelle science : "la philologie comparée qui révèle toutes les lois merveilleuses qui ont présidé à la formation des langues et, avec l'étude et l'observation, nous puiserons à cette source [...]; nous déduirons ainsi à notre tour d'autres lois qui, mieux que n'importe quel texte de grammaire français, aideront à aplanir les difficultés que les Français arrivent rarement à surmonter " ( $L a$ lingua francese, I, 1, $1^{\text {er }}$ décembre $1883: 3$ ).

Ghiotti reste donc fidèle à la méthode traditionnelle "grammaire-traduction ", une méthode classique, nationale qui prend «des couleurs patriotiques» (Pellandra 2001 : 27). comme le souligne Carla Pellandra; en outre, même si la législation scolaire de l'Italie recommande un enseignement pratique d'une langue utile, « les enseignants - et les manuels qu'ils éditent en témoignent - ont tendance à mimer l'enseignement 
prestigieux des langues nobles comme le latin et le grec, des langues qui ne sont pas bassement utilitaires, désintéressés" (ibid.), des langues qui font appel au raisonnement et qui forment l'esprit.

19 Les auteurs de cette revue affrontent également un problème très important pour l'époque, à savoir la formation des professeurs de langue et notamment ceux de français. Comme ils le rappellent dans le $\mathrm{n}^{\circ} 15 \mathrm{du} 1^{\mathrm{er}}$ juillet 1884, la loi du 15 novembre 1859 qui régit encore l'instruction secondaire a classé les enseignants de français en trois catégories : incaricati, reggenti et titolari des trois classes des écoles techniques. Il s'agissait d'une bonne disposition à l'époque car les enseignants n'étaient pas assez nombreux et ne pouvaient satisfaire toutes les demandes, donc c'était une sorte d'invitation pour les jeunes gens à se consacrer à l'étude et à l'enseignement du français. Mais on a accepté des personnes qui n'avaient aucune préparation et les écoles publiques sont remplies de professeurs incompétents. Il fallait, remarquent-ils, qu'à ces propositions correspondent des examens garantissant le savoir et les capacités didactiques du futur professeur. Or, ce n'est pas le cas: les examens ne sont pas en mesure de donner ces garanties car aucun des documents demandés pour l'admission ne prouve la culture générale du candidat et le diplôme obtenu, c'est-à-dire l'habilitation, le brevet pour enseigner coûte trop peu d'efforts à certains. Le titre perd donc de sa valeur.

D'ailleurs, un licencié en lettres et une personne ayant 18 ans et possédant une attestation de bonne conduite peuvent se présenter avec les mêmes droits; puis les rares difficultés des examens d'habilitation offrent les mêmes probabilités de réussite à celui qui est «nourri de bonnes études philologiques " qu'à celui qui connaît peu l'italien et sait seulement quelques rudiments de français et ignore tout des principes de la philologie comparée et des disciplines pédagogiques » (La lingua francese, I, 15, $1^{\mathrm{er}}$ juillet $1884: 261$ ). En outre, les examens sont différents d'une ville à l'autre ; il est donc évident que tant que durera cette situation, « le diplôme de capacité cessera d'être pour les écoles publiques une garantie de la valeur didactique et littéraire de qui est appelé à y enseigner le français » (ibid.). Ghiotti et Dogliani vont proposer une réforme de cet examen sur laquelle je ne m'attarde pas, mais il faut signaler que la revue défend avant tout une préparation "classique » des professeurs qui devront avoir un bon niveau d'études philologiques.

\section{Les critiques de Romeo Lovera}

21 Romeo Lovera qui fonde en janvier 1894 une autre revue, le Bollettino di Filologia Moderna, une revue qui est envoyée gratuitement aux professeurs de français des écoles secondaires, s'insurge contre les vieilles méthodes d'enseignement basées essentiellement sur la comparaison entre la langue maternelle et la langue étrangère. "L'étude analytique des analogies entre notre langue et l'idiome étranger - que les programmes imposent au cours des premières années d'enseignement et que l'on pourrait faire plus tard [...] - est un bagage inutile et même gênant ", déclare-t-il (Bollettino di Filologia Moderna, III, 1, 31 janvier 1901: 4. C'est moi qui traduis). D'après lui, en effet, on peut faire la comparaison entre les deux langues uniquement « lorsqu'il y a déjà une parfaite connaissance de la langue étrangère » car la grammaire comparée ne représente que la dernière étape «aussi bien chronologiquement que philologiquement » (ibid.) sur le chemin de la linguistique. 

Italie, en fondant des écoles et des instituts techniques, devait se mettre à l'œuvre avec des critères modernes » souligne $R$. Lovera dans le premier numéro de son périodique (ibid., I, 1, 15 janvier 1894 : 3) ; l'instruction technique est une instruction populaire, il faut donc penser aux résultats pratiques. Or, les programmes sont « un amoncellement mal organisé de règles, de préceptes, d'injonctions qui ne font pas honneur aux rédacteurs » (ibid.). Quel est l'objectif de l'enseignement des langues se demande-t-il ? C'est de conduire l'élève à écrire et à parler dans la langue qu'il apprend. Il constate que le programme de la 1 ère année de l'école technique ne s'occupe que de la prononciation et de l'orthographe, les élèves n'apprennent donc rien de la langue parlée, ils ne savent pas s'exprimer. Il parle aux élèves sur un ton polémique mais, en réalité, c'est aux enseignants qu'il s'adresse indirectement lorsqu'il déclare : « vous ne savez aucun mot, nous ne savez rien demander, [...] vous saurez peut-être m'expliquer l'imbroglio Si ces six scies-ci, scient si bien, scie ces syx [sic] cyprès-ci; mais vous ne savez pas dire que vous avez faim et que vous voulez manger ». La conclusion de Lovera est très simple : après un an de français, les élèves en savent moins qu'un «docker qui sait au moins comprendre et se faire comprendre » (ibid., I, 1, 15 janvier $1894: 2$ ).

Donc, si les programmes voulaient donner autant d'importance à la grammaire en $1^{\text {ère }}$ année, ils pouvaient introduire l'étude des verbes réguliers, l'élève pourrait ainsi former des phrases avec les mots qui sont présentés dans la nomenclature à la fin de chaque leçon. C'est une erreur d'attendre la fin de la $3^{\mathrm{e}}$ année d'enseignement pour parler le français; " dès la $1^{\text {ère }}$ année, après quelques mois d'école, il faut faire des exercices de conversation, avec des questions et des réponses, avec la répétition de passages de lecture, faciles, concernant les activités courantes de la vie». Le but de l'apprentissage d'une langue n'est pas de faire de l'élève un grammairien (" un grammatico ») mais au contraire de le rendre « apte à lutter avec de bonnes armes dans les situations de cette vie, si agitée et si rapide " (ibid.: 3$)^{8}$.

Lovera propose donc d'adopter les nouvelles méthodes : celle de Börner ou la méthode directe ou encore celle de Gouin ; les professeurs pourront adopter l'une ou l'autre ou même les fondre. De toute façon elles ont toutes une base commune: ce sont les principes qui guident l'association de phonétique dont le président Viëtor est le plus ardent défenseur d'une réforme de l'enseignement des langues étrangères. Quelles sont les tendances générales du programme de cette association? Tout d'abord, enseigner la langue parlée et non pas la langue archaïque de la littérature ; familiariser les élèves avec les sons de la langue étrangère en utilisant l'alphabet phonétique dans la première partie du cours ${ }^{9}$; étudier les phrases et les expressions les plus usuelles de la langue étrangère ; enseigner la grammaire d'une manière inductive, comme " un corollaire et une généralisation des faits observés durant la lecture »(ibid., III, 1, 31 janvier 1901 : 8).

Lovera constate qu'une fois sortis de l'enfance, il est difficile de penser dans la langue étrangère et aucune grammaire, aucun dictionnaire, aucune traduction (version ou thème) ne peuvent donner cette faculté aux élèves. C'est la raison pour laquelle il faut se libérer de la langue maternelle parce que la traduction introduit des complications inutiles dans l'acte de parole. En 1901, dans le $n^{\circ}$ 6-7 de sa revue, Lovera souligne qu'aujourd'hui, les professeurs se sont rendu compte qu'en utilisant seulement «la méthode grammaticale on n'arrive à rien » (ibid., III, 6-7, 30 juin-31 juillet 1901 : 93) ; ils ont donc commencé à étudier la langue vivante, celle que l'on parle pour des raisons commerciales ou pour les relations avec des personnes étrangères. Prenant la défense

Documents pour l'histoire du français langue étrangère ou seconde, 35 | 2005 
de la nouvelle méthode qui «est scientifique parce qu'elle est basée sur les lois naturelles ", Lovera tire à boulets rouges sur l'ancienne, la méthode classique, laquelle avait « peut-être sa raison d'être pour les langues mortes » mais qui, aujourd'hui, «se réduit à une simple compilation de règles grammaticales, données comme autant d'apophtegmes $»^{10}$.

Contrairement à Ghiotti qui préconisait l'utilisation du dictionnaire pour faire les exercices de traduction, Lovera cite une lettre de Berge d'Embrun adressée à Damiano Ricci - auteur d'un ouvrage d'exercices, une lettre dont le rédacteur approuve le contenu. L'auteur de cette lettre constate que la «recherche des mots dans le vocabulaire [...] demande beaucoup de temps, engendre de l'ennui, du dégoût, fatigue excessivement les jeunes gens, leur rend fastidieuse une étude que l'on devrait, au contraire, chercher à rendre agréable autant que possible, et ne présente pas une utilité correspondante à la fatigue, car tout le monde sait qu'il ne suffit pas de chercher une fois seule un mot dans le vocabulaire pour le graver dans l'esprit ». D'après lui, comme d'après Lovera, une méthode pour l'enseignement des langues sera d'autant plus rationnelle qu'elle se rapprochera de la méthode que suit la nature, c'est pourquoi il condamne « ceux qui font apprendre à leurs pauvres élèves, pendant la première année d'étude, des listes interminables d'homonymes et de noms dont le genre n'est pas le même dans les deux langues » (ibid., I, 9, 30 juin $1894: 2$ ). Il déclare également que l'on a exagéré outre mesure l'analogie entre le français et l'italien; en effet, "pour le petit enfant, pour le commençant, cette analogie ne sert à rien, n'est d'aucune aide, parce que l'enfant ignorant de tout ne peut pas la distinguer » (ibid. : 3). En d'autres termes et pour résumer le concept qui sert de guide à Lovera et à sa revue, il met en évidence le besoin d'études pratiques, des études qui préparent les élèves à la vie professionnelle.

Que vont faire les professeurs? Adopteront-ils la méthode raisonnée, comparative de Ghiotti ou la méthode directe de Lovera? En regardant les changements opérés dans les programmes ministériels, les nombreuses éditions de la grammaire, les très nombreuses réimpressions du dictionnaire du premier (une nouvelle édition sera publiée en 1960), il est impossible de nier l'importance de ce professeur qui a formé des générations d'étudiants et qui peut apparaître comme un nouveau Goudar.

\section{BIBLIOGRAPHIE}

\section{Sources primaires}

GHIOTTI, Candido. 1868. Grammatica ragionata della lingua francese. Nuovo corso di lezioni teoricopratiche dettate col confronto della Lingua Italiana e coll'Analisi Filologica. Alessandria : P. Ragazzone.

- 1875. Grammatica ragionata della lingua francese. Torino : Petrini.

- 1877. Le nouveau Morand. Dialogues italiens-français adaptés aux formes et aux sujets de la moderne conversation et enrichis d'exercices préliminaires de nomenclature méthodique et de phraséologie d'un Vocabulaire Géographique et de Noms propres de personnes. Turin : Imprimerie Roux et Favale. 
- 1884. Grammatica ragionata della lingua franceseNuovo corso di lezioni teorico-pratiche dettate col confronto della lingua italiana e con ragionamenti filologico. Torino, Milano, Firenze : Paravia.

- 1898. Compendio della grammatica ragionata delle lingua francese. Unidicesima edizione. Torino : Libreria G.B. Petrini.

GHIOTTI, C., DOGLIANI, G. 1886. La nomenclature en action. Exercices élémentaires de conversation précédés d'un traité de prononciation à l'usage des élèves des écoles techniques. Torino : Petrini.

- 1890. Recueil d'exercices et de thèmes pour servir d'application aux règles de grammaire et de syntaxe. Torino : Petrini.

- 1891. Grammatica ragionata e storica della lingue francese Lezioni dettate col confronto delle lingue italiana e latina ad uso degli Istituti d'istruzione secondaria classica e tecnica e degli aspiranti al Diploma di abilitazione all'insegnamento. V edizione. Torino-Roma-Milano-Firenze-Napoli : Paravia.

- 1894. Mosaïque littéraire Livre de lecture à l'usage des écoles secondaires d'Italie. Torino : Petrini.

- 1905. La correspondance d'une maison de commerce en France et en Italie / La corrispondenza di una casa commerciale in Italia e in Francia. Torino : Petrini.

\section{Revues}

Bollettino di Filologia moderna, Anno I, 1894 ; Anno III, 1901 ; Anno IV, 1902 ; Anno V, 1903 ; Anno VI, 1904.

La lingua francese, Anno I-Anno VII, 1883-1890.

\section{Sources secondaires}

BIVONA, Rosalia. 2001. «'L'Italie est faite, il faut faire les Italiens' : la construction de l'identité nationale dans les manuels scolaires ", in Marie-Christine KOK ESCALLE, Francine MELKA, dir. Changements politiques et statut des langues. Histoire et épistémologie 1780-1945. Amsterdam-Atlanta : Rodopi. 215-233.

FERRETTI, Ange. 1874. De l'enseignement des langues étrangères. Reggio dans l'Émilie : Imprimerie d'Étienne Calderini.

MINERVA, Nadia, PELLANDRA, Carla dir. 1997. Insegnare il francese in Italia. Repertorio analitico di manuali pubblicati dal 1625 al 1860.Bologna : CLUEB.

PELLANDRA, Carla. 1991. « Professeurs de français d'autrefois. Analyse de la revue La lingua francese nelle scuole secondarie d'Italia de 1883 à 1892 ». Documents 8. 221-228.

PELLANDRA, C. 1992. « Les livres d'un professeur de français d'autrefois : le fonds Ferretti du Dipartimento di Lingue e Letterature Straniere Moderne de Bologne ». Documents 9. 24-29.

PELLANDRA, C. 1993. «Le débat sur la méthode directe en Italie ». Études de Linguistique Appliquée 90, avril-juin : « Pour et contre la méthode directe. Historique du mouvement de réforme de l'enseignement des langues de 1880 à 1914 ». 40-49.

PELLANDRA, C. 2001. « La diffusion du français dans le bassin méditerranéen de 1880 à 1914 ». Documents 27. 11-32.

PUREN, Christian. 1988. Histoire des méthodologies de l'enseignement des langues, Paris : Nathan CLE International. 


\section{NOTES}

1. Archives d'État, Rome, Ministero Pubblica Istruzione, Personale 1860-1880, Busta 987.

2. Cette loi est celle que le ministre du Royaume de Sardaigne avait présentée en 1859 pour fondre après la deuxième guerre d'Indépendance les systèmes scolaires piémontais et lombards (cf. Pellandra $1991: 225$ ).

3. Cf. Pellandra $1993: 40-49 ; 2001: 26-27$.

4. Certaines phrases des exercices de traduction refléteront même ces idées car j'ai relevé, par exemple, la phrase suivante : « Lo studio delle lingue straniere deve seguire lo studio della lingua nazionale », L'étude des langues étrangères doit suivre l'étude de la langue nationale (1875 : 97).

5. Il s'agit par exemple de l'ouvrage de François-Joseph Noël et de Charles-Pierre Chapsal intitulé Nouvelle grammaire française sur un plan très-méthodique avec de nombreux exercices d'orthographe, de syntaxe et de ponctuation, Milan, Sonzogno, édité en 1851 ou de celui de Charles-François Lhomond, Éléments de la grammaire française, Milan, François Colombo, édité en 1854.

6. Ghiotti $1868: 3$. Ce cahier, écrit-il, était divisé en 14 chapitres : Prononciation, Déclinaison, Pluriels, Adjectifs, Comparaisons, Pronoms, Verbes, Participes, Particules, Syntaxe, Orthographe, Phraséologie, Lexique, Application.

7. Archives d'État, Rome, Ministero Pubblica Istruzione, Personale 1860-1880, Busta 987.

8. Romeo Lovera qui signera quelques articles sous l'anagramme Omero Valero s'opposera également à la méthode d'ollendorff, une méthode qui se voudrait naturelle mais qui n'est que « la castration la plus insipide de la pensée et l'interprétation la plus maladroite du principe qu'il faut apprendre une langue étrangère comme on apprend la langue maternelle » (ibid., III, 1, 31 janvier 1901 : 6). Cf. également Pellandra $1992: 28$.

9. Lovera fera connaître Passy dans sa revue et sera un ardent défenseur de la phonétique.

10. Ibid., III, 8, 31 octobre 1901 ; c'est dans ce numéro que Romeo Lovera publie l'article « Il metodo grammaticale-traduttivo ed il metodo intuitivo "; il reprendra le même article, mais cette fois en français dans le numéro suivant sous le titre plus explicite «Critique de l'ancienne méthode classique » (IV, 3-4, mars-avril 1902).

\section{RÉSUMÉS}

Cette étude se propose de retracer la figure de Candido Ghiotti. Ce professeur de français, auteur de nombreux ouvrages (dont son célèbre dizionario scolastico bilingue) se fait connaître en 1868 en publiant une grammaire $\mathrm{du}$ français proposant une nouvelle approche de l'étude et de l'apprentissage de la langue : « il metodo filologico comparato », une méthode raisonnée fondée sur la comparaison et l'analogie entre l'italien et le français; elle sera contestée par Romeo Lovera, défenseur de la méthode directe. Néanmoins Ghiotti reste une figure emblématique du professeur de français en Italie.

This study proposes retracing the figure of Candido Ghiotti. This teacher of French, the author of numerous works (including his famous bilingual dizionario scolastico), became known in 1868 when he published a French grammar proposing a new approach to the study and learning of the language: "il metodo filologico comparato", a reasoned method based on a comparison and analogy between Italian and French: it was questioned by Romeo Lovera, the defender of the direct method. However Ghiotti remains an emblematic figure of a French professor in Italy. 
INDEX

Mots-clés : Ghiotti, grammaire du français, méthode raisonnée, apprentissage de la langue

Keywords: Ghiotti, French grammar, reasoned method, learning of the language

\section{AUTEUR}

\section{MARIE-FRANCE MERGER}

Université de Pise 